BULLETIN OF THE

AMERICAN MATHEMATICAL SOCIETY

Volume 78, Number 5, September 1972

\title{
FIBER PRESERVING CELLULAR DECOMPOSITIONS
}

\author{
BY JOHN J. WALSH ${ }^{1}$
}

Communicated by Steve Armentrout, April 3, 1972

1. Introduction. Let $(Y, P, B)$ be a locally trivial bundle with fiber $M^{r}(r \neq 4)$, a compact connected metric manifold with or without boundary; furthermore, assume $B$ is a finite-dimensional, locally compact metric space. Let $G=\{G[b] \mid b \in B\}^{*}$ be an upper semicontinuous (usc) decomposition of $Y$ with each $G[b]$ a cellular decomposition of $P^{-1}(b)$ such that $P^{-1}(b) / G[b] \approx M$. (Let $A$ be a collection of sets; then $A^{*}$ denotes the union of the sets in $A$.) In this note, we prove that $P^{*}: Y / G \rightarrow B$, defined by $P^{*}\left(P^{-1}(b) / G[b]\right)=b$, is a locally trivial bundle with fiber $M$. The special cases where $M$ is an $i$-sphere $(i=1,2,3)$ have been proved in [1] and [2].

The work of Dyer and Hamstrom on completely regular mappings in [1] and [3], that of Kirby and Edwards on local contractibility of spaces of homeomorphism of manifolds in [4] and that of Siebenmann on approximating cellular maps by homeomorphisms in [5] are all essential for the proof of the main result.

2. Terminology. All spaces are assumed to be separable metric. A set $K$ in the interior of an r-manifold is cellular if $K=\bigcap\{J[n] \mid n=1,2, \ldots\}$ where each $J[n]$ is topologically an $r$-cell and $J[n+1]$ is in the interior of $J[n]$. A set $K$ in the boundary of an r-manifold is cellular if it is cellular as a subset of the boundary. A cellular decomposition of a manifold is an usc decomposition in which each element is cellular.

REMARK 1. Using this definition of cellular, it is necessary that each decomposition element be entirely in the interior or entirely in the boundary of the manifold; however, this restriction can be avoided by working in the more general setting of cell-like decompositions. The proof in this paper holds in this latter setting for $r \neq 3,4$; but for simplicity, we give only the definition of cellular (see [5]).

A map $f: X \rightarrow Y$ is completely regular provided that, given $\varepsilon>0$ and $y \in Y$, there is a $\delta>0$ such that for each point $y^{\prime}$ within $\delta$ of $y$, there exists a homeomorphism from $f^{-1}\left(y^{\prime}\right)$ onto $f^{-1}(y)$ which moves no point as much as $\varepsilon$. A triple $(X, f, Y)$ is a locally trivial bundle with fiber $F$ if, for each $y \in Y$, there is an open set $U$ containing $y$ and a homeomorphism $\phi: U \times F \rightarrow f^{-1}(U)$ with $f \phi=\pi$ where $\pi: U \times F \rightarrow U$ is the projection.

AMS 1970 subject classifications. Primary 54B15, 57A60; Secondary 54C10.

${ }^{1}$ This research was supported in part by National Science Foundation Grant GP-19589. 


\section{Main Theorem.}

THEOREM. Let $B$ be a finite-dimensional compact metric space and let $M^{r}$ be a compact connected $r$-manifold $(r \neq 4)$. Let $G=\{G[b] \mid b \in B\}^{*}$ be an usc decomposition of $M \times B$ with each $G[b]$ a cellular decomposition of $M \times\{b\}$ for which $M \times\{b\} / G[b] \approx M$. If $r=5$, assume that no nondegenerate element of $G[b]$ meets the boundary of $M \times\{b\}$. If $P: M \times B / G \rightarrow B$ is defined by $P(M \times\{b\} / G[b])=b$, then $(M \times B / G, P, B)$ is a locally trivial bundle with fiber $M$.

Proof. Let $\pi: M \times B \rightarrow M \times B / G$ be the mapping induced by the decomposition $G$ and let $\pi[b]=\pi \mid M \times\{b\}$. For $b$ and $b^{\prime}$ in $B$, let $T\left[b, b^{\prime}\right]: M \times\{b\} \rightarrow M \times\left\{b^{\prime}\right\}$ be defined by $T\left[b, b^{\prime}\right]((m, b))=\left(m, b^{\prime}\right)$; furthermore, assume the metric on $M \times B$ is such that $T\left[b, b^{\prime}\right]$ moves no point more than the distance of $b$ from $b^{\prime}$. We shall use $D($,$) to denote$ all metrics.

The mapping $P$ is completely regular. Let $\varepsilon>0$ be given; choose $0<\delta$ $<\varepsilon / 3$ such that $D\left((m, b),\left(m^{\prime}, b^{\prime}\right)\right)<\delta$ implies that $D\left(\pi(m, b), \pi\left(m^{\prime}, b^{\prime}\right)\right)$ $<\varepsilon / 3$. Using [5, Theorem A], for each $b \in B$, there is a homeomorphism $\Sigma[b]: M \times\{b\} \rightarrow M \times\{b\} / G[b]$ such that $D(\Sigma[b]((m, b)), \pi[b]((m, b)))$ $<\varepsilon / 3$ for all $m \in M$. If $D\left(b, b^{\prime}\right)<\delta$, define a homeomorphism $g=\Sigma\left[b^{\prime}\right]$ $\circ T\left[b, b^{\prime}\right] \circ \Sigma[b]^{-1}$ from $P^{-1}(b)$ onto $P^{-1}\left(b^{\prime}\right)$. Applying the triang.e inequality twice, it is easily shown that $g$ moves no point as much as $\varepsilon$.

Since the space of homeomorphisms of $M$ is locally contractible, see [4, Corollary 1.1], it follows immediately from [1] or [2, p. 242] that $(M \times B / G, P, B)$ is a locally trivial bundle.

REMARK 2. If $r=4$, the theorem remains true if it can be shown that each $\pi[b]$ can be approximated by homeomorphisms.

REMARK 3. Is it true that $(M \times B / G, P, B)$ is a trivial bundle? This will be true if $B$ is contractible [6, p. 53].

REMARK 4. By applying the above theorem locally, we can now prove the theorem stated in the introduction. If, in addition to the hypothesis of this latter theorem, $B$ is a compact connected $s$-manifold, then $Y / G$ is a manifold and, if $r+s \neq 4, Y / G \approx Y$ [5, Theorem $\mathrm{A}]$. (If $r=5$, it must be assumed that no nondegenerate element of $G[b]$ meets the boundary of $P^{-1}(b)$.)

4. A decomposition theorem for $E^{n}$. Let $S^{n}$ denote the $n$-sphere and view the $n$-dimensional euclidean space $E^{n}$ as $S^{n}$ minus a point.

Lemma. Let $G$ be a cellular decomposition of $E^{n}=S^{n}-\{t\}$ with $E^{n} / G \approx E^{n}(n \neq 4)$. Then $G^{\prime}=G \cup\{t\}$ is a cellular decomposition of $S^{n}$ with $S^{n} / G^{\prime} \approx S^{n}$ and, if $\pi: S^{n} \rightarrow S^{n} / G^{\prime}$ is the mapping induced by the decomposition, for each $\varepsilon>0$, there is a homeomorphism $\Sigma: S^{n} \rightarrow S^{n}$ with $D(\Sigma(x), \pi(x))<\varepsilon$ for all $x \in S^{n}$ and $\Sigma(t)=t$. 
Proof. The only interesting part of this lemma is that $\pi$ can be approximated by homeomorphisms leaving $t$ fixed; and this follows easily from [5, Theorem A] as follows. Let $\mathscr{E}: S^{n}-\{t\} \rightarrow(0, \varepsilon)$ be a continuous function with the property that $\mathscr{E}(x)$ approaches zero as $x$ approaches $t$. By [5, Theorem A], there is a homeomorphism $\Sigma^{\prime}: S^{n}-\{t\} \rightarrow S^{n}-\{t\}$ with $D\left(\pi(x), \Sigma^{\prime}(x)\right)<\mathscr{E}(x)$ for all $x \in S^{n}-\{t\}$. $\Sigma$ is obtained by letting $\Sigma=\Sigma^{\prime}$ on $S^{n}-\{t\}$ and letting $\Sigma(t)=t$.

The following corollary generalizes results in $[1 ; p=2, q=1]$ and $[3 ; p=3, q=1]$.

CoRollary. Let $p+q=n(p \neq 4)$ and let $G=\left\{G[x] \mid x \in E^{q}\right\}^{*}$ be a usc decomposition of $E^{p} \times E^{q}$ with each $G[x]$ a cellular decomposition of $E^{p} \times\{x\}$ for which $E^{p} \times\{x\} / G[x] \approx E^{p}$. Then $E^{p} \times E^{q} / G \approx E^{p} \times E^{q}$.

OUTLINE OF PROOF. Consider $E^{p} \times E^{q} \subseteq S^{p} \times E^{q}$ with $S^{p} \times E^{q}-E^{p}$ $\times E^{q}=\{t\} \times E^{q}$. Extend each $G[x]$ to $G^{\prime}[x]=G[x] \cup\{(t, x)\}$ and let $G^{\prime}=\left\{G^{\prime}[x] \mid x \in E^{q}\right\}^{*}$. Let $P^{\prime}: S^{p} \times E^{q} / G^{\prime} \rightarrow E^{q}$ be defined by $P^{\prime}\left(S^{p} \times\{x\} / G^{\prime}[x]\right)=x$ and let $P=P^{\prime} \mid E^{p} \times E^{q} / G$. Using the lemma together with the fact that the space of homeomorphisms of $S^{n}$ leaving a point fixed is locally contractible [4], it can be proved that, for each $x \in E^{q}$, there is an open set $U$ containing $x$ and a homeomorphism $\phi: S^{p} \times U \rightarrow P^{\prime-1}(U)$ with $\phi\left(S^{p} \times\left\{x^{\prime}\right\}\right)=S^{p} \times\left\{x^{\prime}\right\} / G[x]$ and $\phi\left(\left(t, x^{\prime}\right)\right)$ $=\left(t, x^{\prime}\right)$ for all $x^{\prime} \in U$. This implies that $\left(E^{p} \times E^{q} / G, P, E^{q}\right)$ is a locally trivial bundle and, since $E^{q}$ is contractible, actually a trivial bundle [6, p. 53]. In particular, $E^{p} \times E^{q} / G \approx E^{p} \times E^{q}$.

\section{REFERENCES}

1. E. Dyer and M.-E. Hamstrom, Completely regular mappings, Fund. Math. 45 (1958), 103-118. MR 19, 1187.

2. M.-E. Hamstrom, Regular mappings: A survey, Proc. First Conf. on Monotone Mappings and Open Mappings (SUNY at Binghamton, Binghamton, N. Y., 1970), State Univ. of New York at Binghamton, Binghamton, N. Y., 1971, pp. 238-254. MR 43 \#1140.

3. - A decomposition theorem for $E^{4}$, Illinois J. Math. 7 (1963), 503-507. MR 27 \# 1928.

4. R. D. Edwards and R. C. Kirby, Deformation of spaces of imbeddings, Ann. of Math. (2) $93(1971)$.

5. L. C. Siebenmann, Approximating cellular maps by homeomorphisms (preliminary version).

6. N. Steenrod, The topology of fibre bundles, Princeton Math. Series, vol. 14, Princeton Univ. Press, Princeton, N. J., 1951. MR 12, 522.

7. S. K. Kim, Local triviality of completely regular mappings, Duke Math. J. 38 (1971).

Department of Mathematics, State University of New York at Binghamton, BINGHAMTON, NEW YORK 13901 